\title{
Metallization of Polyimide Films with Enlarged Area by Conducting the Catalyzation in Supercritical Carbon Dioxide
}

Tso-Fu Mark Chang ${ }^{1,2, a}$, Cheng-Chuan Wang ${ }^{3, b}$, Chia-Ying Yen ${ }^{3}$, Shin-Hwa Chen ${ }^{3}$, Chun-Yi Chen ${ }^{1,2}$, and Masato Sone $e^{1,2}$

${ }^{1}$ Precision and Intelligence Laboratory, Tokyo Institute of Technology 4259-R2-35 Nagatsuta-cho Midori-ku Yokohama 226-8503 Japan

${ }^{2}$ CREST, Japan Science and Technology Agency 4259 Nagatsuta-cho, Midori-ku, Yokohama 226-8503, Japan

${ }^{3}$ Material and Chemical Research Laboratories, Industrial Technology Research Institute No. 195, Chung Hsing Rd., Sec. 4, Chutung, Hsinchu 31040, Taiwan

*Corresponding Author.

ae-mail: chang.m.aa@m.titech.ac.jp (Mark Chang)

be-mail: chengchuan@itri.org.tw (Cheng-Chuan Wang) 


\begin{abstract}
This study reports the metallization of polyimide films with enlarged area by conducting the catalyzation step in supercritical $\mathrm{CO}_{2}$. Defect-free Ni coating with high adhesion strength on the polyimide film having dimensions of $12.0 \times 4.5 \mathrm{~cm}^{2}$ was realized using a high pressure vessel with diameter and height of only 4.0 and $4.5 \mathrm{~cm}$, respectively. The ability to conduct the catalyzation of polyimide films having size larger than size of the reaction cell is a result of the enhanced transport properties caused by the supercritical $\mathrm{CO}_{2}$. With 8 min of plating time, the Ni coating thickness on the polyimide film reached $1.077 \mu \mathrm{m}$, and the resistivity and adhesion strength were $1.4 \Omega$ and $1.5 \mathrm{MPa}$, respectively.
\end{abstract}

Keywords: Metallization, Polyimide Film, Supercritical Carbon Dioxide, Catalyzation, Electroless

Plating, Nickel 


\section{Introduction:}

The flexible and biocompatible properties of polymers make polymers as one of the promising materials for the next generation of micro-electro-mechanical systems (MEMS). In fact, bio-medical micro-devices composed of polymer components such as micro-blood checkers and strain gauges embedded into a soft contact lens have already been realized [1,2]. Among the polymers to be applied in MEMS, polyimide (PI) has a lots of outstanding properties, such as good thermal stability, low dielectric constants, inherent surface inertness and low dissipation factors. These properties make PI one of the popular polymers to be applied in MEMS technology [3,4]. However, polymers, such as PI, often have very low electrical conductivity, which limits the applications in MEMS. Therefore, metallization of the polymers to increase electrical conductivity of the polymeric materials is required for the applications as MEMS components.

For metallization of the polymers, electroless plating is the most commonly used technique because of its simplicity and the ability to deposit uniform metallic coatings on complicated-shape surfaces [5]. In previous studies, properties of the metallic coatings deposited on the polymer surface are reported to be improved by using supercritical $\mathrm{CO}_{2}\left(\mathrm{sc}-\mathrm{CO}_{2}\right)$ [6-12]. $\mathrm{Sc}-\mathrm{CO}_{2}$ is $\mathrm{CO}_{2}$ at temperature and pressure higher than the critical point. Critical point of $\mathrm{CO}_{2}$ is $31.1{ }^{\circ} \mathrm{C}$ and $7.39 \mathrm{MPa}$. The enhancement is suggested to be results of the extremely low surface tension and the non-polar properties. For metallization by the electroless plating technique, a catalyzation step is needed. When sc- $\mathrm{CO}_{2}$ is applied 
in the catalyzation step and a metal organo complex is used as the source of the metal catalyst, impregnation of the metal catalyst deep into surface of the polymeric substrate is observed [8,9]. After impregnation of the metal catalyst into the polymer surface, reduction of the metal ions in the electroless plating step would be initiated from the metal catalyst to form metallic-root-like structure under the polymer surface to anchor the metallic coatings on the polymer surface. These metallic roots are expected to be extremely effective in improving adhesion strength of the metallic coatings on the polymer film similar to the root system of plants.

However, size of the polymeric substrates catalyzed in the sc- $\mathrm{CO}_{2}$ is usually small. Because of the high pressure needed to form the sc- $\mathrm{CO}_{2}$, the catalyzation process has to be conducted in a high pressure vessel. Typical volume of the high pressure vessel is usually small, such as $50 \sim 70 \mathrm{ml}$ with dimensions ranged from $4 \sim 5 \mathrm{~cm}[6-9,12]$, to lower the cost of the entire high pressure apparatus. In order to ensure proper mixing of the metal organo complex sc- $\mathrm{CO}_{2}$, PI films catalyzed in the high pressure vessel usually have dimensions in half of the dimension of the high pressure vessel, such as $1.0 \times 2.0 \mathrm{~cm}^{2}$. The dimensions are too small in the practical applications. Therefore, it is necessary to develop a method to increase dimensions of the PI films catalyzed with the $\mathrm{sc}-\mathrm{CO}_{2}$ and maintains a relatively small size of the high pressure vessel at the same time to improve the feasibility of the $\mathrm{sc}-\mathrm{CO}_{2}$-assisted catalyzation technique for the practical applications in MEMS. 


\section{Experimental Section:}

Details of the high pressure apparatus used in this study could be found in a previous study [7]. The PI films used in this study were Kapton ${ }^{\circledR}$ PI films with dimensions of $14.0 \times 4.5 \mathrm{~cm}^{2}$. The pressurized vessel was made of $316 \mathrm{~L}$ stainless steel with cylindrical-shaped inner space of $4.0 \mathrm{~cm}$ in diameter and $4.5 \mathrm{~cm}$ in height as shown in Fig. 1. The metallization of the PI films were divided into two steps, which are the catalyzation step and the electroless plating step. In the catalyzation step, $2 \mathrm{mg}$ of $\mathrm{Pd}$ (II) acetylacetonate $\left(\operatorname{Pd}(\mathrm{acac})_{2}\right)$ was added into the high pressure vessel. Excess amount of the $\operatorname{Pd}(\mathrm{acac})_{2}$ was used to ensure the concentration to be always close to the saturation concentration in the catalyzation step [12]. The $\mathrm{Pd}(\mathrm{acac})_{2}$ was purchased from Sigma-Aldrich ${ }^{\circledR}$ with a purity of $99 \%$, and the $\mathrm{CO}_{2}$ was provided by Nippon Tansan Gas with a minimum purity of $99.99 \%$. Length of the PI films used in the study was much larger than both the diameter and height of the high pressure vessel. Thus, the PI film was first rolled along the long side as shown in Fig. 1 then it could be placed into the high pressure vessel directly. The pressure, temperature, and catalyzation time were $15 \mathrm{MPa}, 80^{\circ} \mathrm{C}$, and $60 \mathrm{~min}$, respectively. This catalyzation step is named sc- $\mathrm{CO}_{2}$ catalyzation (SCC). After the SCC, conventional electroless plating at ambient pressure and $70{ }^{\circ} \mathrm{C}$ with a Ni electrolyte was conducted for 3 to 8 min to give Ni coatings with different thickness. The Ni electrolyte was commercially available electrolyte,

NICHEM PF 500 (containing $5.5 \mathrm{~g} / \mathrm{L}$ of $\mathrm{Ni}^{2+}$ and $\mathrm{pH}=4.7$ ), purchased from Atotech Taiwan Ltd. 
The Ni coating thickness was determined by observing cross-sectional image of the films using a field emission scanning electron microscope (Fe-SEM, S-4500SE, Hitachi High technologies Co., Ltd). Resistivity of the Ni coated PI film was evaluated by a four point probe meter (Loresta EP Model MCP-T360, Mitsubishi Chemical Analytech Co., Ltd.). For each Ni coated PI film, the resistivity evaluation was conducted at three locations, and the evaluation was conducted three times at each location. One location was at the mid-point of the Ni coated area, and rest of the two locations were both $3 \mathrm{~cm}$ away from the mid-point along the long side of the film. In a total of 9 data points were collected and averaged for each Ni coated PI film. Adhesion strength of the Ni coating on the PI film was evaluated by an adhesion puller tester (PosiTest AT, DeFelsko Corp.). Adhesion strength tests were conducted at the three locations mentioned in the resistivity evaluation section hence three data points were collected and averaged for the adhesion strength tests.

\section{Results and Discussion:}

Impregnation of the Pd into the PI takes place from the interface between the PI and the Pd-contained solution. Rolling the PI film raised a concern that the overlapped part of the PI surface might not have enough contact time with the Pd-contained sc- $\mathrm{CO}_{2}$ to have uniform Pd catalyzation of the entire PI surface. However, viscosity of the sc- $\mathrm{CO}_{2}$ is very low $\left(32.488 \mu \mathrm{Pa} \cdot \mathrm{s}\right.$ at $80{ }^{\circ} \mathrm{C}$ and $15 \mathrm{MPa}$ [13]), and the 
surface tension is technically zero. Wetting of the overlapped PI surface with the Pd-contained sc- $\mathrm{CO}_{2}$ and transfer of the Pd ions to the surface of the overlapped PI surface are expected to be high. Thus, uniform catalyzation of the entire PI surface including the overlapped part could be achieved by the SCC. The complete and uniform catalyzation was confirmed in the image shown in Fig. 2, where $12.0 \times 4.5$ $\mathrm{cm}^{2}$ of the surface was completely covered by bright metallic Ni. Size of the PI film was $14.0 \times 4.5 \mathrm{~cm}^{2}$. $2 \mathrm{~cm}$ of the length was sacrificed to be used to position the PI film in the Ni electrolyte in the plating step. Therefore, only $12 \mathrm{~cm}$ long of the PI film was immersed in the Ni electrolyte.

Morphology of the Ni coatings on the PI films is shown in Fig. 3. Some particles were observed on the Ni coatings. Size and numbers of the particles were found to increase with an increase in the plating time. On the other hand, no cracks and defects were observed on the Ni coatings obtained in this study, shown in the SEM images. Defects are often results of the un-uniform catalyzation, and cracks are caused by the difference between the mechanical properties of $\mathrm{Ni}$ and PI. The results further demonstrated that rolling the PI film in the SCC step would not cause any problems to give defect-free and uniform $\mathrm{Ni}$ coatings because of the improved transfer efficiency provided by the sc- $\mathrm{CO}_{2}$.

Thickness of the Ni coating reached $0.628 \mu \mathrm{m}$ in 3 min of the electroless plating. Relationship between the Ni coating thickness and the plating time is shown in Fig. 4. Generally, the Ni coating thickness increased linearly with an increase in the plating time. The resistivity of the Ni coating on the 
PI film was found to be lowered with an increase in the Ni coating thickness as shown in Fig. 5. This result is expected since resistivity has an inverse relationship with cross-sectional area of the electrical conductive layer, which is the Ni coating in this case. With an increase in the Ni coating thickness, the cross-sectional area is increased and leads to the reduction of the resistivity.

The adhesion strengths of the Ni coating on the PI film were all higher than 1.0 MPa as shown in Fig. 6. A decrease in the adhesion strength was observed with an increase in the Ni coating thickness. The decrease in the adhesion strength is suggested to be caused by the increase in the internal stress of the $\mathrm{Ni}$ coatings with an increase in the Ni coating thickness [14].

\section{Conclusions:}

Metallization of $12.0 \times 4.5 \mathrm{~cm}^{2}$ PI films by conducting the catalyzation step in Pd-contained supercritical $\mathrm{CO}_{2}$ using a high pressure vessel with dimensions of $4.0 \mathrm{~cm}$ in diameter and $4.5 \mathrm{~cm}$ in height was demonstrated in this study. The resistivity was found to increase with an increase in the $\mathrm{Ni}$ coating thickness. Although the PI films were rolled in order to be placed in the high pressure vessel in the SCC step, but the adhesion strengths of all of the samples were all larger than 1.0 MPa, even when the $\mathrm{Ni}$ coating thickness exceeded $1 \mu \mathrm{m}$. The results demonstrated that the $\mathrm{sc}-\mathrm{CO}_{2}$ catalyzation technique could be applied to metallize PI films with an enlarged area while using a high pressure vessel 
with much smaller dimensions. This finding is promising for practical application of the $\mathrm{sc}-\mathrm{CO}_{2}$ catalyzation technique in the applications of MEMS.

\section{Acknowledgment:}

This work is supported by CREST Project operated by the Japan Science and Technology Agency (JST) and The Ministry of Economic Affairs in Taiwan. 


\section{References:}

[1] M. Leonardi, P. Leuenberger, D. Bertrand, A. Bertsch, P. Renaud, First steps toward noninvasive intraocular pressure monitoring with a sensing contact lens, Invest. Ophthalmol. Vis. Sci. 45 (2004) 3113-3117.

[2] M. Palacio, B. Bhushan, N. Ferrell, D. Hansford, Nanomechanical characterization of polymer beam structures for BioMEMS applications, Sens. Actuators A 135 (2007) 637-650.

[3] S.Y. Xiao, L.F. Che, X.X. Li, X.L. Wang, A novel fabrication process of MEMS devices on polyimide flexible substrates, Microelectron. Eng. 85 (2008) 452-457.

[4] S. Petroni, G. Maruccio, F. Guido, M. Amato, A. Campa, A. Passaseo, M.T. Todaro, M. De Vittorio, Flexible piezoelectric cantilevers fabricated on polyimide substrate, Microelectron. Eng. 98 (2012) 603-606.

[5] M. Charbonnier, M. Romand, Polymer pretreatments for enhanced adhesion of metals deposited by the electroless process, Int. J. Adhes. Adhes. 23 (2003) 277-285.

[6] B.H. Woo, M. Sone, A. Shibata, C. Ishiyama, K. Masuda, M. Yamagata, Y. Higo, Metallization on polymer via quantitatively controlled catalyzation in sc- $\mathrm{CO}_{2}$ and electroless plating with sc- $\mathrm{CO}_{2}$ emulsion for micro and nano-device, Microelectron. Eng. 86 (2009) 1179-1182. 
[7] B.H. Woo, M. Sone, A. Shibata, C. Ishiyama, K. Masuda, M. Yamagata, Y. Higo, Effects of Sc- $-\mathrm{CO}_{2}$ catalyzation in metallization on polymer by electroless plating, Surf. Coat. Technol. 203 (2009) 1971-1978.

[8] B.H. Woo, M. Sone, A. Shibata, C. Ishiyama, K. Masuda, M. Yamagata, S. Edo, T. Hatsuzawa, Y. Higo, Metallization on polymer by catalyzation in supercritical $\mathrm{CO}_{2}$ and electroless plating in dense $\mathrm{CO}_{2}$ emulsion, Surf. Coat. Technol. 202 (2008) 3921-3926.

[9] H. Adachi, K. Taki, S. Nagamine, A. Yusa, M. Ohshima, Supercritical carbon dioxide assisted electroless plating on thermoplastic polymers, J. Supercrit. Fluids 49 (2009) 265-270.

[10] D.P. Long, J.M. Blackburn, J.J. Watkins, Chemical fluid deposition: A hybrid technique for low-temperature metallization, Adv. Mater. 12 (2000) 913-915.

[11] P.J. Tsai, C.H. Yang, W.C. Hsu, W.T. Tsai, J.K. Chang, Enhancing hydrogen storage on carbon nanotubes via hybrid chemical etching and Pt decoration employing supercritical carbon dioxide fluid, Int. J. Hydrogen Energy, 37 (2012) 6714-6720.

[12] S. Yoda, A. Hasegawa, H. Suda, Y. Uchimaru, K. Haraya, T. Tsuji, K. Otake, Preparation of a platinum and palladium/polyimide nanocomposite film as a precursor of metal-doped carbon molecular sieve membrane via supercritical impregnation, Chem. Mater. 16 (2004) 2363-2368.

[13] NIST Reference Fluid Thermodynamic and Transport Properties Database. 
[14] K. Parker, H. Shah, The stress of electroless nickel deposits on beryllium, J. Electrochem. Soc. 117 (1970) 1091-1094. 


\section{List of Figures:}

Figure 1. Process flow for supercritical $\mathrm{CO}_{2}$ catalyzation of the large-area PI film.

Figure 2. The PI films after the electroless plating step by a plating time of (a) $3 \mathrm{~min}$, (b) $5 \mathrm{~min}$, (c) 6 min, and (d) 8 min. Dimensions of the plated area are about $12.0 \times 4.5 \mathrm{~cm}^{2}$.

Figure 3. SEM images showing morphology of the Ni coatings obtained with a plating time of (a) 3 min, (b) $5 \mathrm{~min}$, (c) $6 \mathrm{~min}$, and (d) $8 \mathrm{~min}$.

Figure 4. Ni coating thickness with different plating time.

Figure 5. Resistivity of the Ni coating with different Ni coating thickness.

Figure 6. Adhesion strength of the Ni coating on the PI film with different Ni coating thickness. 


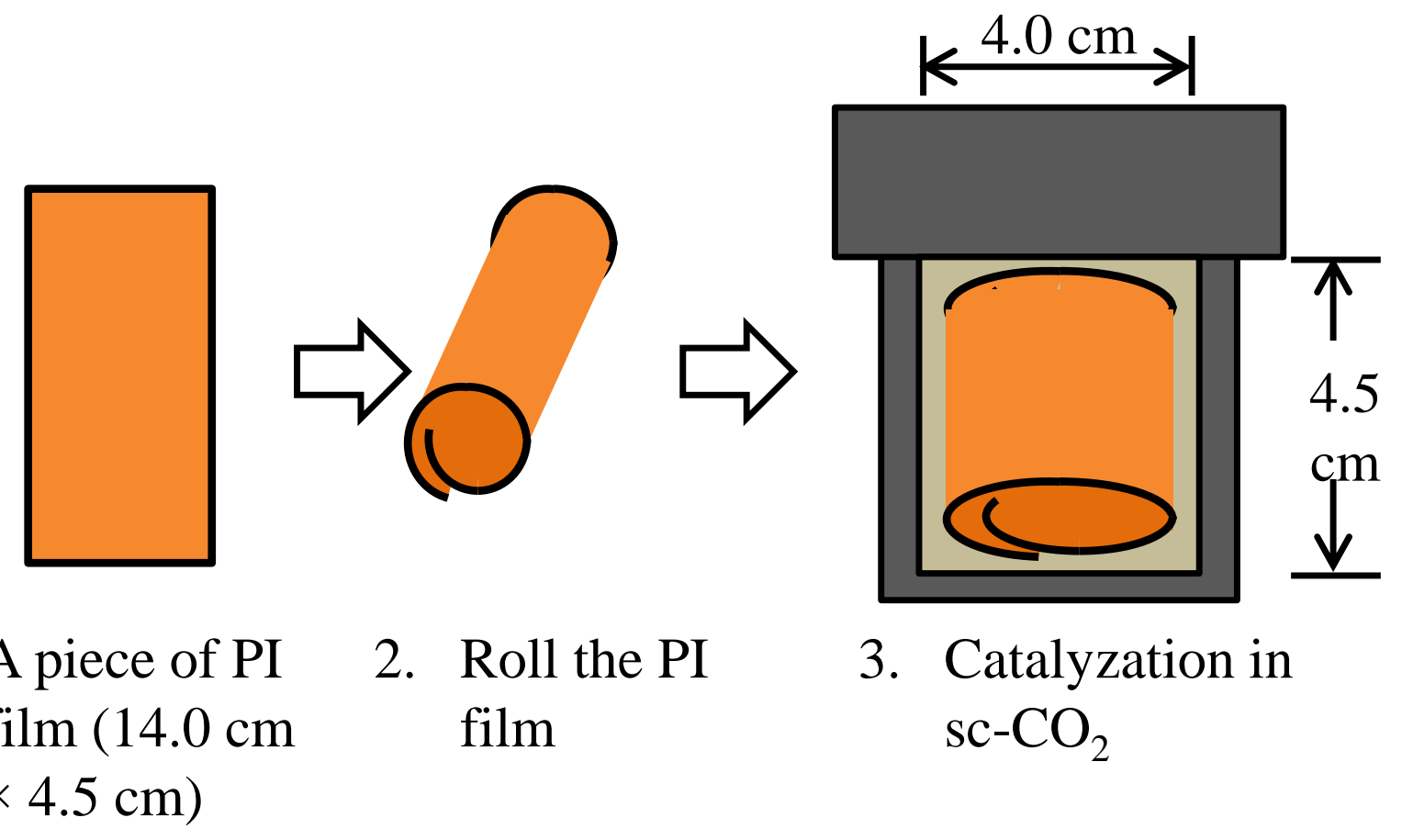

Fig. 1 Process flow for supercritical $\mathrm{CO}_{2}$ catalyzation of the large-area PI film.

1. A piece of PI 2. Roll the PI film $(14.0 \mathrm{~cm}$ $\times 4.5 \mathrm{~cm})$ sc- $\mathrm{CO}_{2}$ 


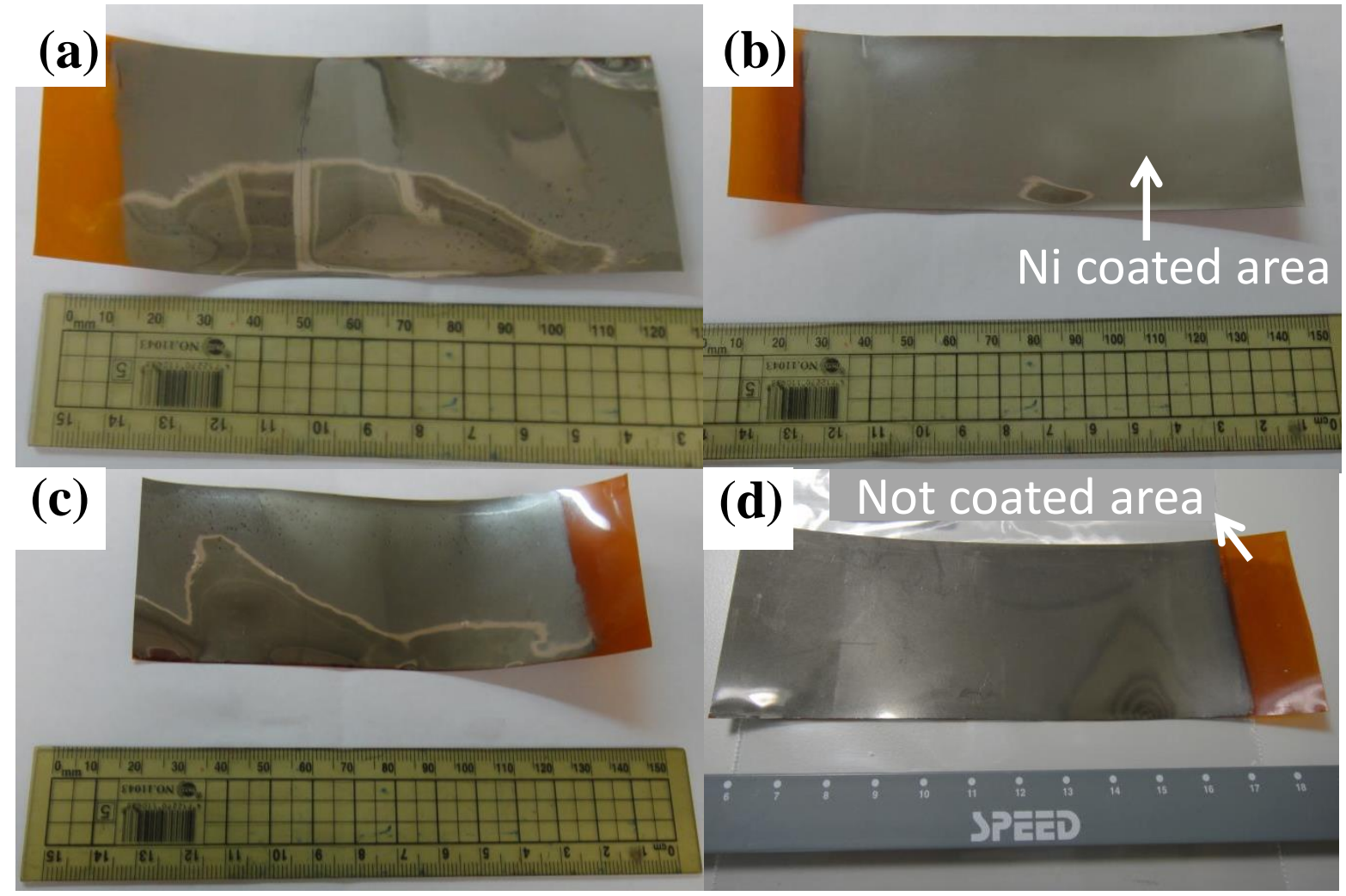

Fig. 2 The PI films after the electroless plating step by a plating time of (a) $3 \mathrm{~min}$, (b) $5 \mathrm{~min}$, (c) $6 \mathrm{~min}$, and (d) $8 \mathrm{~min}$. Dimensions of the plated area are about $12.0 \times$ $4.5 \mathrm{~cm}^{2}$. 


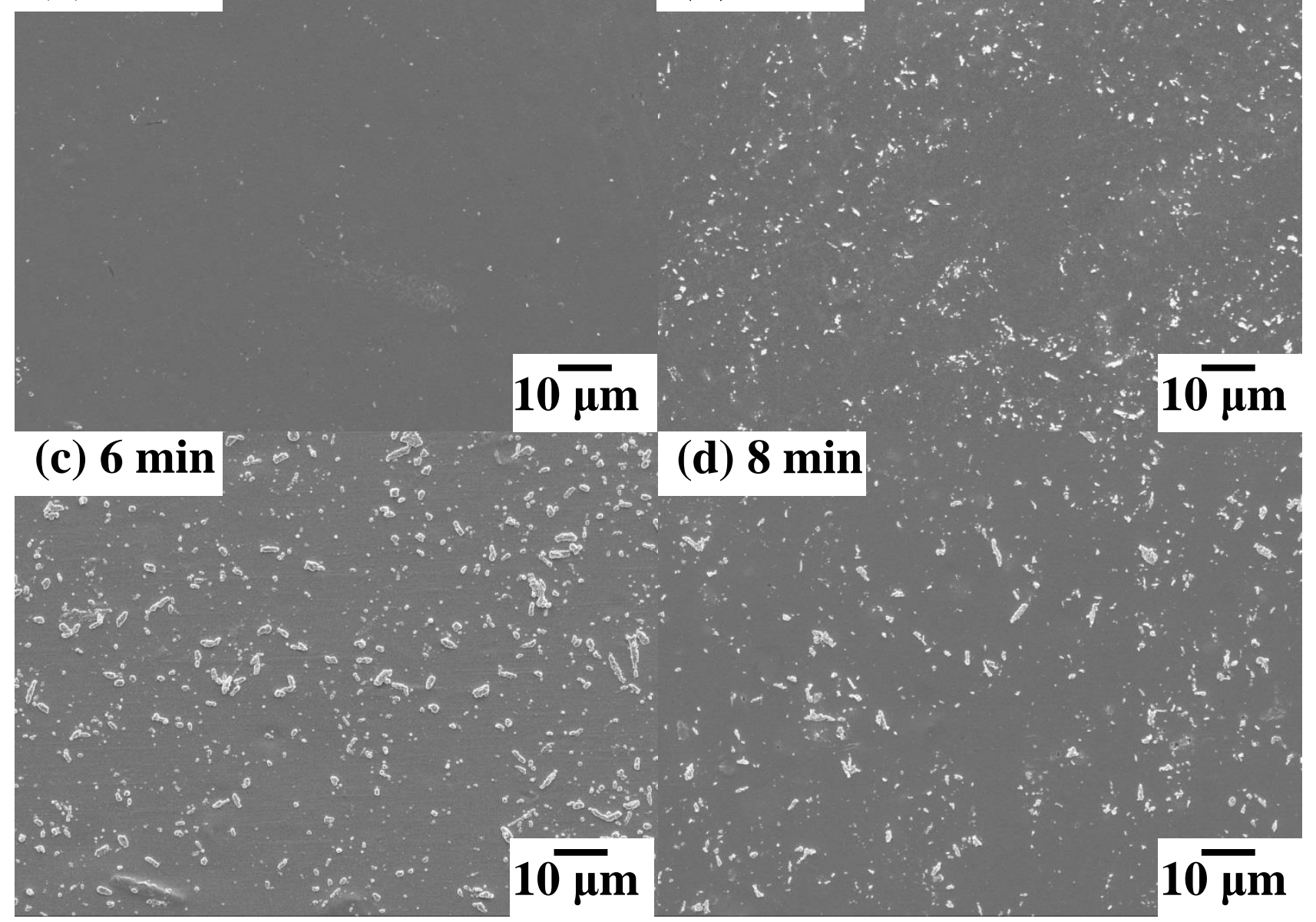

Fig. 3 SEM images showing morphology of the Ni coatings obtained with a plating time of (a) 3 min, (b) 5 min, (c) 6 min, and (d) 8 min. 


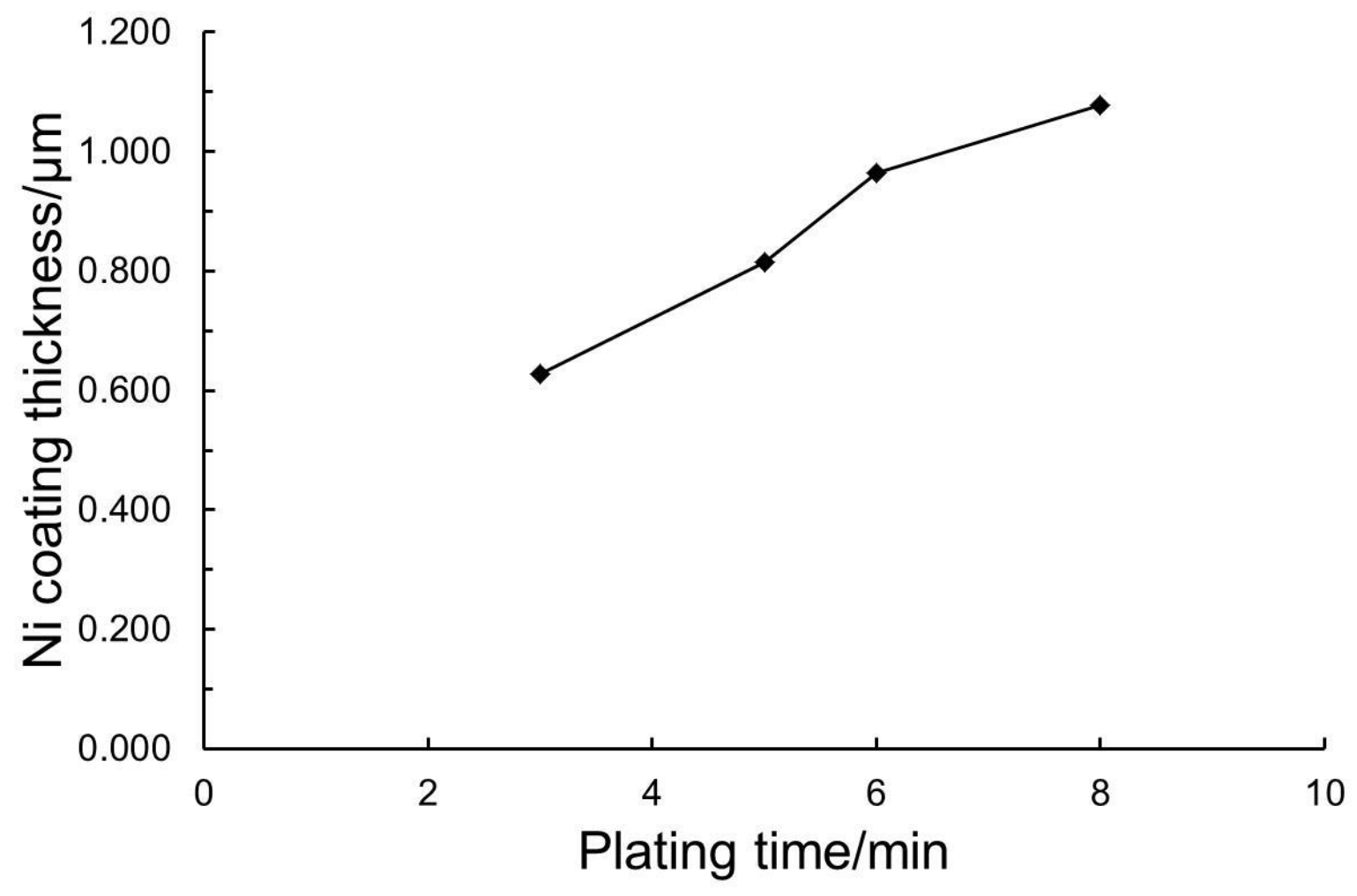

Fig. 4 Ni coating thickness with different plating time. 


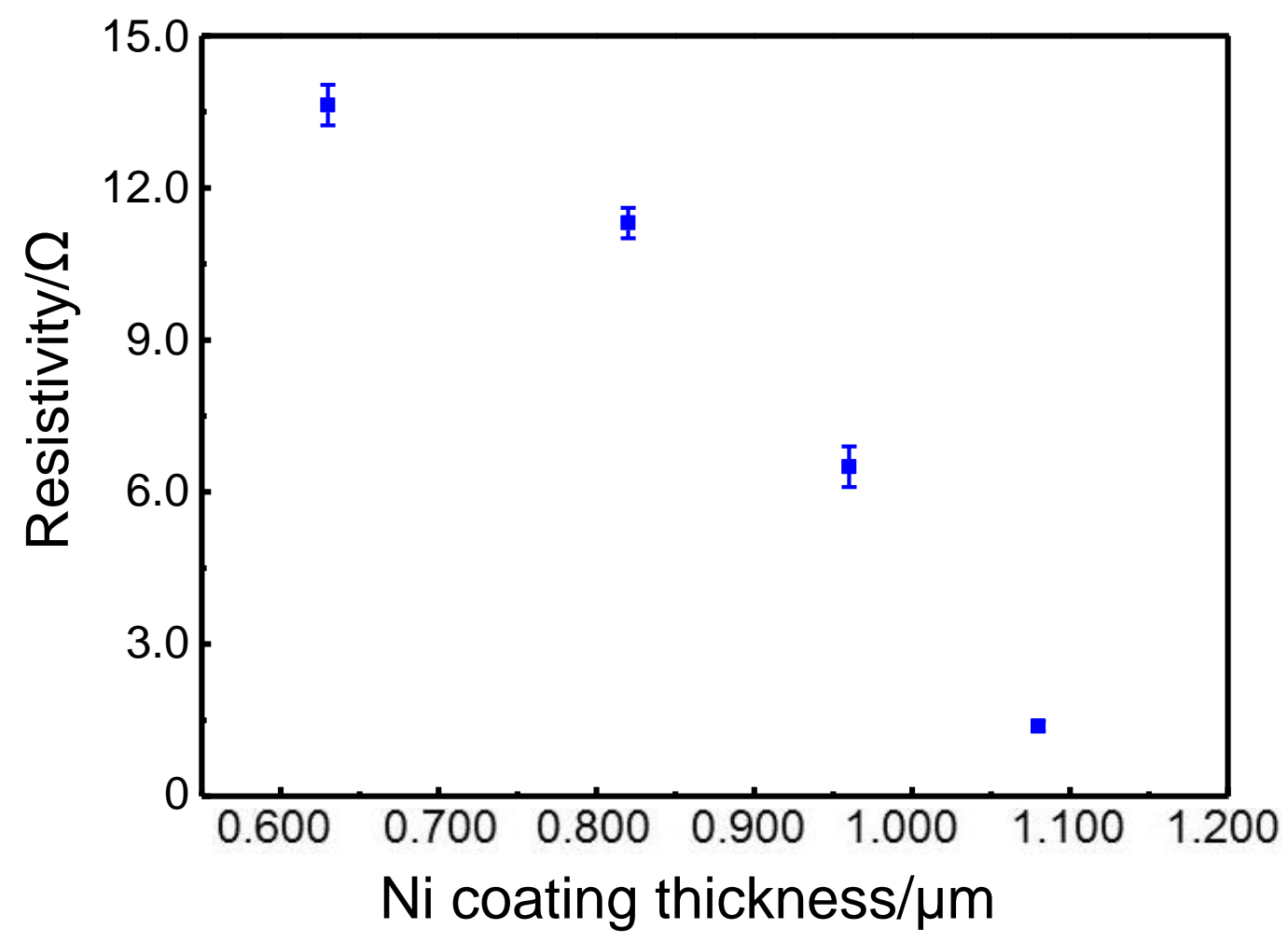

Fig. 5 Resistivity of the Ni coating with different Ni coating thickness. 


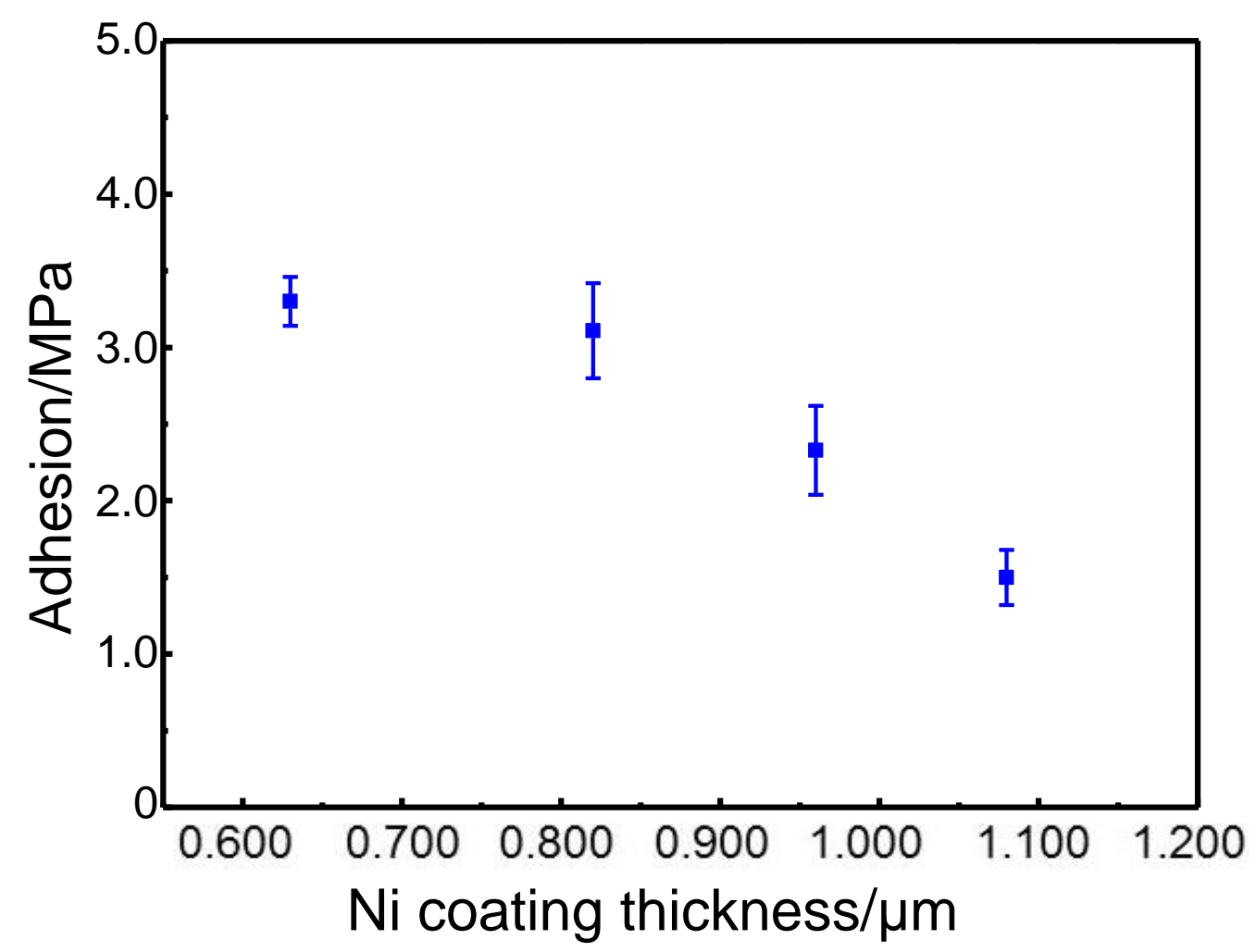

Fig. 6 Adhesion strength of the Ni coating on the PI film with different Ni coating thickness. 
